\title{
AT HIGH ENERGIES
}

\author{
H. $\left.\operatorname{Satz}^{*}\right)$ \\ CERN - Geneva
}

\section{$A B S T R A C T$}

The statistical bootstrap condition for hadronic systems at rest implies an asymptotic density of states increasing exponentially in the total energy. We show that if for a given system the squared energies of all possible subsystems are constrained to lie on linear Regge trajectories, such a behaviour of the level density indeed follows. This result is then used to clarify common features of dual and statistical bootstrap approaches.

*) On leave from the Department of Theoretical Physics, University of Bielefeld, Germany.

Ref.TH. 1649-CERN

5 April 1973 
According to the statistical bootstrap hypothesis 1),2), a hadronic system at rest ("fireball") will decay in cascade fashion into other hadronic systems of the same nature, until finally stable particles are reached as energetically lowest possible fireballs. More precisely, a given fireball is characterized by a level density $\tau\left(\mathbb{M}^{2}\right)$, where $\tau\left(\mathbb{M}^{2}\right) d \mathbb{M}^{2}$ denotes the number of available states in the interval $\mathrm{dM}^{2}$ around the squared mass, $\mathbb{M}^{2}$, of the fireball. The bootstrap proposal now states that $\tau\left(x^{2}\right)$ is the same function for all fireballs, those out of which our given system arose by decay and those into which it in turn will decay. This requirement may be written in the form 2)-4)

$$
\tau\left(P^{2}\right)=\delta_{0}\left(P^{2}-m^{2}\right)+\sum_{\ell=0}^{\infty} \frac{B^{\ell}}{\ell !} \int \prod_{i=1}^{\ell}\left\{d^{4} k_{i} \tau\left(k_{i}^{2}\right)\right\} \delta^{(4)}\left(\sum_{i=1}^{\ell} k_{i}-P\right)
$$

with

$$
\begin{aligned}
& \delta_{0}\left(x^{2}\right)=\theta\left(x_{0}\right) \delta\left(x^{2}-m^{2}\right) \\
& \tau\left(x^{2}\right)=\theta\left(x^{2}\right) \theta\left(x_{0}-m\right) \tilde{\tau}\left(x^{2}\right)
\end{aligned}
$$

For simplicity, we consider here a world with only one kind of scalar particles of mass $m$ and obeying Boltzmann statistics. The statistical bootstrap equation (1) represents a determining condition for the energy behaviour of the number of states per mass interval. For large $\mathbb{M}$, its solution becomes 1 ), 3), 5)

$$
\tau\left(M^{2}\right) \simeq \text { const. } * M^{-3} e^{M / T_{0}} \quad \text { i } P^{2}=M^{2}
$$

with $\mathrm{T}_{0}$ determined by

$$
2 \pi m B T_{0} K_{1}\left(\frac{m}{T_{0}}\right)=2 \ln 2-1
$$

[At the point $T=T_{0}$ the grand partition function

$$
Z(T)=\int d^{4} P e^{-\beta P} \tau\left(P^{2}\right) \quad \text { i } \beta^{2}=T^{-2}
$$

becomes singular 4),5).] Thus we know that unless the number of states per energy interval increases linearly exponential in the energy, the condition (1) cannot be satisfied. 
This leads us naturally to ask : for what kind of systems does the density of allowed states increase in such a fashion? The density of points in phase space

$$
g\left(M^{2}\right)=\sum_{N=2}^{\infty} \frac{\lambda^{N}}{N !} \int \prod_{i=1}^{N} \frac{d^{3} n_{i}}{2 p_{i 0}} \delta^{(4)}\left(\sum_{i=1}^{N} n_{i}-P\right)
$$

which corresponds to the number of states for a free hadron gas in a given interaction region 6) $\lambda$, is in fact knowr to behave asymptotically as 7)

$$
g\left(M^{2}\right)=\text { const. } * M^{-4}\left(\lambda M^{2}\right)^{2 / 3} e^{3\left[\lambda \pi M^{2} / 2\right]^{1 / 3}}
$$

and thus increases less than linearly exponential. Moreover, the essence of this result is not altered by the introduction of factorizable polynomial weights in momentum space :

$$
\frac{d^{3} n_{i}}{2 n_{i 0}} \rightarrow \frac{d^{3} n_{i}}{2 n_{i 0}}\left(p_{i 0}\right)^{\nu}
$$

The new form resulting from (8) yields for $\nu \neq 0$ different energy exponents in $g\left(M^{2}\right)$ than that of (7) ; but these exponents always increase less than linearly in $\mathbb{M}$. Thus a considerable modification is necessary to achieve the bootstrap increase (3).

The aim of this note is to show that in fact one obtains such a form in a world in which all states of positive erergy hadronic systems are constrained to integral values of usual Regge trajectories, i.e., trajectories linear in the squared mass $\mathbb{M}^{2}=\mathrm{s}$.

$$
\alpha(s)=\alpha_{0}+\alpha^{\prime} s
$$

of the system in question. To understand this, let us go back one step before Eq. (6). The probability of obtaining an $\mathrm{N}$ particle final state from a given initial state $\mathrm{P}^{2}=\mathbb{M}^{2}$ is determined by

$$
g_{N}\left(p^{2}\right)=\frac{1}{N !} \int \prod_{i=1}^{N} \frac{d^{3} n_{i}}{2 \mu_{i 0}} \delta^{(4)}\left(\sum_{i=1}^{N} n_{i}-p\right)\left|\left\langle n_{1} \cdots n_{N}|V| P\right\rangle\right|^{2}
$$


where the transition matrix element contains all dynamics. Assuming equidistribution in momentum space

$$
\left|\left\langle n_{1} \cdots n_{N}|V| P\right\rangle\right|^{2}=\lambda^{N}
$$

yields (6); the ratio of two different final states is then determined by their relative phase space volumes, i.e., by the relative number of detailed configurations, or states, compatible with the given final state information.

To obtain a form more suitable both for comparison with the cascade decay of the bootstrap and for the introduction of a Regge quantization, let us rewrite (10) in terms of invariant subenergies $w_{i}$, where

$$
W_{i}^{2}=\left(\sum_{\ell=1}^{i+1} p_{i}\right)^{2} \quad i \quad i=1,2, \cdots, N-1
$$

This can be accomplished by iterating the relation $\left.{ }^{8}\right)$

$$
\frac{d^{3} n_{1}}{2 r_{10}} \frac{d^{3} n_{2}}{2 p_{20}}=d W \frac{d^{3} P}{2 P_{0}} d^{2} \Omega \frac{K}{2}
$$

where $W$ denotes the rest mass, $\vec{P}$ the linear momentum of the (1-2)

system ; $K$ is the magnitude, $\Omega$ the angular orientation of the momentum of either of the two particles in the (1-2) centre-of-mass, with

$$
K=\frac{1}{2 W}\left[\left(W^{2}-\left(m_{1}+m_{2}\right)^{2}\right)\left(W^{2}-\left(m_{1}-m_{2}\right)^{2}\right)\right]^{1 / 2}
$$

As a result of iterating (13) and performing the delta function integration we then have 9)

$$
g_{N}\left(p^{2}\right)=\frac{1}{N !} \frac{1}{2 M} \int \prod_{i=1}^{N-2} d W_{i} \int_{i=1}^{N-1} d^{2} \Omega_{i}\left\{\prod_{i=1}^{N-1} \frac{K_{i}}{2}|\langle|\cdots N| V \mid P\rangle|^{2}\right\}
$$

where the angular integration goes over $4 \pi$, while the invariant subenergies are restricted by

$$
\begin{aligned}
& W_{k-1}+m \leq W_{k} \leq M-(N-k-1) m ; k=1, \cdots, N-1 \\
& W_{0} \equiv m \quad i \quad W_{N-1} \equiv M
\end{aligned}
$$


If we now assume (11), we of course recover (6) and the $\mathbb{M}^{2 / 3}$ increase of $\ln g\left(\mathbb{M}^{2}\right)$. The situation changes, however, if we take an equidistribution not in the space of $\left\{w_{i}\right\}$, but one in $\left\{w_{i}^{2}\right\}$ instead, i.e., if we count a constant number of states per unit of $W_{i}^{2}$ rather than per unit of $W_{i} \cdot$ With

$$
\left\{\prod_{i=1}^{N-1} \frac{K_{i}}{2 W_{i}}|\langle|\cdots N| V \mid P\rangle|^{2}=r^{N-1}\right.
$$

we obtain (taking for simplicity $m=0$ )

$$
\begin{aligned}
g_{N}\left(M^{2}\right) & =r\left(\frac{r}{2}\right)^{N-2} \int \prod_{i=1}^{N-2} d W_{i}^{2} \int_{i=1}^{N-1} d^{2} \Omega_{i} \\
& =4 \pi r\left(2 \pi r M^{2}\right)^{N-2} /(N-2) !
\end{aligned}
$$

which yields

$$
g\left(M^{2}\right)=\frac{2}{M^{2}} I_{2}\left(2 \sqrt{2 \pi r M^{2}}\right)
$$

and hence asymptotically in $\mathbb{M}$

$$
g\left(M^{2}\right) \simeq \text { const. } * M^{-5 / 2} e^{2 \sqrt{2 \pi r} M}
$$

a linearly exponential form. Thus an equidistribution over all squared subenergies, $W_{i}^{2}$, as required by a Regge description for all subsystems, leads to a phase space volume, or total number of states, increasing linearly exponential in the energy.

To make the result more transparent, it may be helpful to consider a simplified case. Let us look at a world of identical onedimensional particles capable of taking on discrete energies

$$
q_{i}=n ; n=0,1, \cdots \quad \text { i } i=1, \cdots, N
$$

The total number of all allowed $N$ particle states at a given over-all energy $M$ then is 


$$
d(M)=\sum_{N=2}^{\infty} \frac{1}{N !}\left[\prod_{i=1}^{N} \sum_{q_{i}=0}^{\infty} \delta\left(\sum_{i=1}^{N} n_{i}, M\right)\right]
$$

The "invariant subenergies"

$$
w_{i}=\sum_{l=1}^{i} q_{i} \quad i \quad i=1,2, \cdots, N \quad \text { i } \quad w_{N}=M
$$

of course also have discrete values

$$
w_{i}=n \quad i \quad n=0,1, \cdots \quad i \quad i=1, \cdots, N
$$

Using (23), the level density $d(M)$ becomes

$$
\begin{aligned}
d(M) & =\sum_{N=2}^{\infty} \frac{1}{N !}\left[\sum_{w_{1}=0}^{M} \sum_{w_{2}=w_{1}}^{M} \cdots \sum_{w_{N-1}=w_{N-2}}^{M}\right] \\
& =\sum_{N=2}^{\infty} \frac{M^{N-1}}{N !(N-1) !}=\frac{1}{\sqrt{M}}\left[I_{1}(2 \sqrt{M})-1\right]
\end{aligned}
$$

which asymptotically gives with

$$
d(M) \simeq \text { const. } M^{-3 / 4} e^{2 \sqrt{M}}
$$

a form increasing exponentially in $\mathbb{M}^{\frac{1}{2}}$. Replacing the quantization of subenergies (24) by one in squared subenergies in accord with the usual Regge description

$$
w_{i}^{2}=n \quad i \quad n=0,1, \cdots \quad i \quad i=1, \cdots, N
$$

yields in a similar fashion

$$
\begin{aligned}
d(M) & =\sum_{N=2}^{\infty} \frac{1}{N !}\left[\sum_{w_{1}^{2}=0}^{M^{2}} \sum_{\omega_{2}^{2}=\sigma_{1}^{2}}^{M^{2}} \cdots \sum_{\omega_{N-1}^{2}=w_{N-2}^{2}}^{M^{2}}\right] \\
& =\frac{1}{M}\left[I_{1}(2 M)-1\right]
\end{aligned}
$$


and hence with

\section{$d(M) \simeq$ const. $* M^{-3 / 2} e^{2 M}$}

a level degeneracy increasing linearly exponential in energy $\mathbb{M}$. It should be emphasized that the agreement between the bootstrap solution and Regge counting holds only for trajectories linear in $s=\mathbb{M}^{2}$; any other power would destroy the linearity of $\ln \mathrm{d}(\mathbb{M})$ in $\mathbb{M}$.

We close with several remarks to indicate some consequences and implications of our result.

1) - With (20) or (29), we have essentially counted the number of states at which an amplitude, fully constructed from narrow resonances, is different from zero. Further dynamical information will of course generally ascribe different weights to different such states, so that the number of states alone normally does not possess direct physical prediction power [cf., however, Refs. 10) and 11)]. It does so only when we further assume the transition matrix element to be a constant 4) in our "Regge space", e.g., when we claim that

$$
g(M)=\sum_{N=2}^{\infty} \frac{\lambda^{N-1}}{N !}\left[\sum_{w_{1}^{2}=0}^{M} \cdots \sum_{w_{N-1}^{2}=w_{N-2}^{2}}^{M}\right]
$$

constructed as (28), corresponds to the transition probability. Such a statistical or equidistribution model 6) in Regge space is then equivalent to the statistical bootstrap model. On the other hand, the statistical bootstrap condition necessarily requires Regge trajectories linear in $\mathrm{M}^{2}$, if the volume of Regge space is to satisfy (3).

2) - The common level degeneracy in statistical bootstrap and dual resonance 12) descriptions appears now rather clear. For the dual resonance model in the narrow resonance approximation, $g\left(\mathbb{M}^{2}\right)$ is simply the number of points at which the amplitude is non-zero. It does not say anything about general production reactions, as $B_{N}$ is not an equidistribution over Regge space ; in contrast to (17), its angular dependence for a scattering process cannot be neglected. (Nevertheless, for certain problems, e.g., decay of a heavy resonance, $B_{N}$ may be well approximated by an equidistribution 11).) For the statistical bootstrap approach, only the level density matters in comparing different final states, and that must, as it does in Regge space, increase linearly exponential in energy. 
3) - In considering a Regge space as in (28), we have essentially geometrized one dynamical feature, the resonance structure. As duality connects resonance structure and transverse momentum restrictions, it is interesting to look for connections between the two in our framework. In doing so, we have to bear in.mind that we have neither touched on questions of longitudinal motion in a bootstrap approach, nor of spin in Regge space. Consider therefore an isotropic version of the uncorrelated jet model 13), applicable in situations with no initial longitudinal motion, e.g., for $e^{+} e^{-} \rightarrow y \rightarrow$ hadrons 14$)$. With an $N$ particle production cross-section

$$
\sigma_{N}\left(M^{2}\right) \sim \frac{1}{N !} \int \prod_{i=1}^{N}\left\{\frac{d^{3} n_{i}}{2 p_{i 0}} e^{-a \mu_{i 0}}\right\} \delta^{(4)}\left(\sum_{i}^{N} p_{i}-P\right)
$$

the inclusive single particle distribution becomes

$$
2 \mu_{0} \frac{d \sigma}{d^{3} p} \equiv F\left(p_{0}\right) \sim e^{-a \mu_{0}}
$$

provided $p_{0}$ is far enough from the kinematic boundary. A statistical model in Regge space yields, using (18) and (20)

$$
\begin{aligned}
F\left(\mu_{0}\right) & \sim\left\{r \frac{\partial}{\partial r} g\left([P-\mu]^{2}\right)\right\} / g\left(P^{2}\right) \\
& \sim e^{-\frac{1}{T_{0}}\left[\sqrt{M\left(M-2 \mu_{0}\right)}-M\right]} \sim e^{-\mu_{0} / T_{0}}
\end{aligned}
$$

with $T_{0}=\frac{1}{2} \sqrt{2} \pi r$. Thus we obtain the same result from Regge space alone (which incorporates resonance structure) as we get from usual phase space plus transverse momentum cut-off. This seems to us a rather novel manifestation of duality.

Stimulating discussions with R. Hagedorn and H.B. Nielsen are gratefully acknowledged. 


\section{REFERENCES}

1) R. Hagedorn - Nuovo Cimento Suppl. 3, 147 (1965).

2) S. Frautschi - Phys.Rev. D3, 2821 (1971).

3) J. Yellin - CERN Preprint TH. 1513 (1972).

4) R. Hagedorn and I. Montvay - CERN Preprint TH. 1610 (1973).

5) W. Nahm - Nuclear Phys. B45, 525 (1972).

6) E. Fermi - Progr.Theor.Phys. (Japan) 1, 570 (1950).

7) H. Satz - Nuovo Cimento 37, 1407 (1965).

8) Cf., e.g. :

H. Pilkuhn - "The Interactions of Hadrons", North Holland Publishing Company, Amsterdam (1967).

9) H. Satz - Fortschr.Phys. 11, 445 (1963).

10) A. Krzywicki - Phys.Rev. 187, 1964 (1969).

11) M.I. Gorenstein et al. - "Dual Resonance Nodel and Statistical Bootstrap", Kiev Preprint ITP 72-168E (1973).

12) S. Fubini and G. Veneziano - Nuovo Cimento 64A, 811 (1969);

K. Bardakçi and S. Mandelstam - Phys.Rev. 184, 1640 ( 1969).

13) L. Van Hove - Revs.Modern Phys. 36, 655 (1964);

A. Krzywicki - Nuovo Cimento 32, 1067 (1964).

14) J.D. Bjorken and S.J. Brodsky - Phys.Rev. D1, 1416 (1970). 